\title{
Cell Library Creation using ALF
}

\author{
Assim Sagahyroon ${ }^{1}$ and Maddu Karunaratne ${ }^{2}$ \\ ${ }^{1}$ American University, Sharjah, UAE \\ 2 University of Pittsburg, Johnstown, Pennsylvania
}

The design of Integrated Circuit (ASICs and SoCs) typically relies on the availability of a library consisting of predefined components called technology cells. Silicon vendors use proprietary formats to describe technology cells and macro modules in conjunction with numerous translators to feed technology library data to Electronic Design Automation (EDA) tools. Multiple grammar formats are used to represent various aspects of the cells in the same technology library, such as behavior for simulation, timing parameters for synthesis, physical data for layout, noise parameters for signal integrity checks, etc. In addition, most of these formats are highly tool-oriented and are not grammatically consistent. In this paper we will discuss the newly adopted IEEE 1603-2003 Advanced Library Format (ALF) standard which eliminates such drawbacks. This standard defines a grammar for accurate and comprehensive modeling of technology libraries and macro modules in order to bridge the growing gap between new design rules and the analysis required for complex high-end IC implementations.

Keywords: ASIC, SoC, Technology Cells, Advanced Library Format (ALF).

\section{Introduction}

Designing integrated circuits (ICs) and System on Chip (SoC) using 0.18-micron and smaller process technologies pose tremendous challenges for design teams. Perhaps the most significant problem with existing design environments is that power, timing, and signal integrity effects are strongly interrelated in the nanometer domain, but conventional point-solution design tools do not have the capability to consider all of these effects and their interrelationships concurrently. For example, the number of silicon failures caused by signal integrity problems is on the rise due to the lack of existing design tools and methodologies that can address these issues effectively [1].
In this deep submicron era, an increasing number of effects that were previously insignificant are becoming first order and can no longer be ignored in present day IC design requirements. The complex, second-order effects for new subwavelength nanometer processes (residence, inductance, crosstalk, leakage, electromigration, etc.) are modeled in abstraction, mostly for the physical layer. This results in additional number of design iterations that may be required in order to fix problems found very late in the design cycle. Therefore it is becoming much more difficult to design in the nanometer era whilst maintaining expected performance, power (static and active), area, design cycle time, manufacturability and cost scaling. These effects need to be dealt with at all levels: technology process, data extraction and library modeling, logic synthesis, circuit design, place and route, clock distribution, verification, and final test and assembly. Therefore, architecting a technology cell library with these effects in mind can help reduce their adverse impact on chip design [2].

Traditionally, design rules and high margins have been used in practice so far in IC designs to protect and to avoid dealing with signal integrity issues such as crosstalk noise, electromigration, hot electron and antenna effects, etc. Therefore, ASIC design flow has been mostly focused on achieving the steps of RTL Design Closure, Functional Verification and Test, and Timing Closure during physical implementation. These goals are almost always achieved in several iterations, from logic synthesis to layout steps. As the semiconductor feature size reduces towards $90 \mathrm{~nm}$ and below, such practices would not help to exploit the efficient use 
of available technology for competitive advantage. Moreover, with supply voltages reducing to one volt or less, voltage drops due to high switching currents would cause unexpected signal delays in critical circuit areas. Such issues need additional point tools to analyze them and safeguard the designs by applying incremental changes during design and physical implementation phases. Unless the analysis and repair capabilities are combined into the same EDA tool, for high-end designs in SoC domain, the iterative process of analysis and repair of signal integrity violations become inefficient. In general, the quality of results depends on the quality of the analysis models that are provided to the tool. A case in point, for signal noise and electromigration, there was no library modeling language available in the industry that could represent the characterization data. ALF (IEEE standard 1603-2003, approved Sept. 2003) is the only standard language available to model such details and essential information into technology cell libraries and macro modules. Other modeling languages, though none of them are IEEE standards, are being enhanced to accommodate such information to be in par with ALF. At present, ALF is the only available industry standard that can describe not only timing, power and noise, but also electromigration, hot electron, antenna and other factors in consistent and comprehensive formats.

\section{IC Design Flow}

In this section, we will briefly describe a typical design flow for ASIC and IP modules. For a comprehensive discussion of this flow, readers are referred to [3].

The design flow begins with a behavioral specification of the design. The specification document can be an elaborate document that includes delay, area and power constraints, and other criteria that might govern the design. At the behavioral level, we are modeling functionality of the design in the form of an input-output model that suppresses the details about gate and physical level implementations. At this stage, the intended input- output relationship can be verified using functional simulation. Next, either a Behavioral Synthesis tool or a human designer transforms the behavioral description into a Register Transfer Level (RTL) description. At this level, the design is described as a dataflow model that will implement the intended digital circuit. That is, this level consists of components and their interconnections. Logic synthesis tools are then used to convert the RTL description to a gate-level netlist. In essence, the gate-level netlist is a technologyindependent description of the circuit in terms of a netlist of standard cells such as gates, flip-flops, latches, and sometimes multiplexors, counters and interconnections between them. The synthesis tool ensures that the generated netlist meets timing, area and power specifications. The process of transforming this generic cell-based logic network into a vendor-specific network is known as library binding or technology mapping. The library contains a set of parameterized technology cells. These cells are usually provided with their physical layout, timing models, behavioral models, etc. The netlist is then input to an automatic Place and Route tool to generate the physical layout. The layout is verified and then fabricated as an IC chip.

\section{Technology Cell Libraries}

Design approaches can generally be classified into custom and semi-custom designs. Compared to the custom design approach, the complexity and cost of designing an IC is greatly reduced in the semi-custom design approach. The semi-custom designs use predesigned technology cells and (much larger) macro modules that are usually optimized, well-designed, and well-characterized. EDA tools are used by designers to choose among the various available cells/modules and to interconnect them to achieve the desired design functionality and performance. Semi-custom designs can further be subdivided into two categories: arraybased design and cell-based design. Arraybased designs use a prefabricated matrix of nonconnected components named sites; these sites are then interconnected to create a circuit with the desired functionality. This paper is mainly concerned with semi-custom design that is cellbased, for a detailed discussion of array-based design, readers can refer to [4]. Cell-based designs use libraries of pre- designed cells. These cells can be characterized and optimized for the different process technologies that the library 
targets. Each cell in the library is typically parameterized in terms of area, delay, and power. In cell-based designs, engineers can use standard cells and/or macro cells. Examples of standard cells are basic logic gates such as inverters, NAND gates and NOR gates. Examples of macro cells include memories and complex datapath components such as adders and multipliers.

Each cell in an ASIC library must contain at least the following information [3]:

- A circuit schematic

- A physical layout

- A behavioral model

- A detailed timing model

- A wire-load model

- A routing model

- A cell icon

- A test strategy

- A power model

An IC designer needs a high-level or behavioral model for each cell to avoid time consuming and detailed simulation, especially at the initial design iterations. On the other hand, for example, a detailed timing model for each cell is needed in order to assess the performance of the overall design. Silicon Foundries, who are the "library providers", strive to provide their customers with accurate and complete characterization of every cell in their library. In order to characterize a cell, the detailed electrical parasitics of cell layout are extracted and the behavior of each library cell is characterized over a range of output loads and input rise/fall times. Some of the parameters tracked during this process are propagation delay, output rise/fall times and peak/average current. The characterization can then be represented as a closed-form equation or in the form of a lookup table of input rise/fall times, output loading, and device characteristics inside the cell. These library elements are typically simulated using different process scenarios and operating conditions to guarantee reliable operation, sufficient design margin and scalability [4]. The sets of operating conditions may specify parameters such as the process, temperature, voltage and $\mathrm{RC}$ tree models. These can be used during synthesis and timing analysis of the design. Before
ALF, contrasting formats had to be used to represent the same technology cells to suit different applications, even from the same EDA vendor. For example: A technology library is described as a logic library and a physical library. An example of such a commercially available library is the Synopsys technology library [5]. This library can be categorized into two libraries: a logic library and a physical library. The logic library contains information relevant only to the synthesis process and is used for synthesis and design optimization. This information includes pin-to-pin timing, area, pin types and power; no physical information is given in the logic library. On the other hand, the physical library contains the physical characteristics of the cell such as physical dimensions of the cell, layer information and orientation of cells.

Cell libraries are evaluated by considering various aspects of library characteristics such as its synthesis efficiency, robustness, portability, usability, quality, timelines, custom support, and cost. In conclusion, cell libraries determine the overall performance of the optimized logic. A well-characterized cell library will result in fast and efficient designs, whereas a poorly designed and characterized library will, no doubt, degrade the performance of the end product.

\section{A Comprehensive Library Format: The Case for ALF}

In the previous section, the authors discussed the role of the library in the design flow and its importance. In this nanometer era, and as the complexity of IC design progresses, one of the main challenges is cell library development. This process has become very critical and often whether to start off on a new design may depend on the availability of an acceptable cell library for that application. Hence, there is an increased need for library accuracy and consistency across design flows [6].

Currently, many large ASIC and semiconductor companies use internally developed formats to describe the technology cells and macro modules in conjunction with numerous translators to feed technology library data to EDA tools [7-8]. Sometimes, multiple formats are used to represent various aspects of the cells in the 


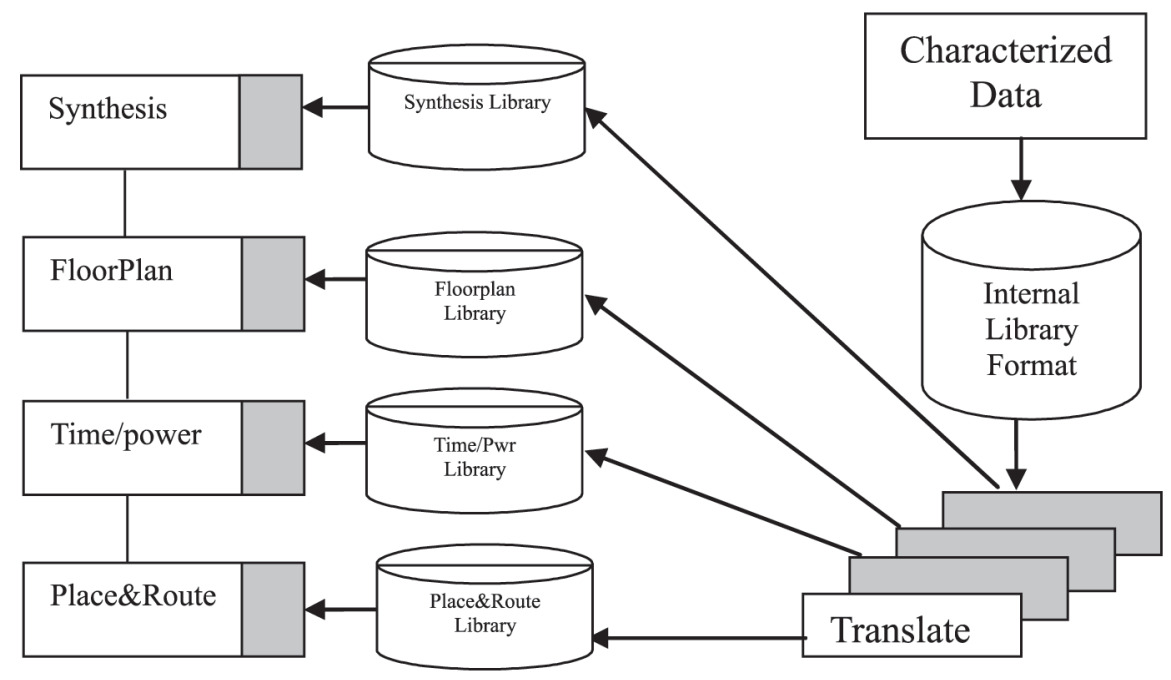

Fig. 1. Current Library Creation Strategies.

same technology library, such as functionality for simulation, timing parameters, physical design characteristics, signal integrity data, etc. Also, most of these formats are highly tooloriented; that is, it is often the case that a silicon vendor company has to generate several different library formats for the same technology family, for different tools to be used in the different parts of the design flows. Figure 1 summarizes the present approach to library creation and usage [9].

To control the complexity of deep submicron design flows and the expensive library creation (repetitive change of tool-specific libraries), an industry-wide standard for library format was needed. The advantages include: reduced cost, improved quality, portability and an appreciable time saving in library creation and validation. The Advanced Library Format (IEEE standard 1603) alleviates the aforementioned problems by providing a modeling language and semantics for the functional, physical, and electrical performance description of technology-specific libraries for cell-based and macro-module-based designs. ALF provides models with functional and performance information including simulation, synthesis, timing analysis, power analysis and signal integrity characteristics. The basic goals of the proposed format can be summarized as follows [10]:

- Simplicity: library creation process must be easy to understand and should not become a cumbersome process.
- Generality: tools of any level of sophistication must be able to retrieve necessary information from the library.

- Expandability: for early adoption and future enhancement possibilities.

- Flexibility: the choice of keeping the information in one library or in separate libraries must be in the hand of the developer; it should not be mandated by the standard.

- Efficiency: the complexity of the design information requires that the process of retrieving information from the library does not become a bottleneck.

- Ease of implementation: unambiguous description and accuracy of contents.

- Acceptance: preference for the new standard library over existing libraries.

ALF was designed to be more general purpose in scope and applicability. Additionally, the need for easy migration from the existing proprietary formats was also taken into consideration early during its development. It, therefore, had to be a superset of all existing and contemplated technology data formats, which it really is. However, it is not a mere collection of different features, but has a solid language structure and highly enriched expressiveness, which would lend itself for many potential and innovative EDA applications. The fundamental purpose of the standard is for ALF to serve as the primary database for all third-party applications of technology cells. One can think of ALF 


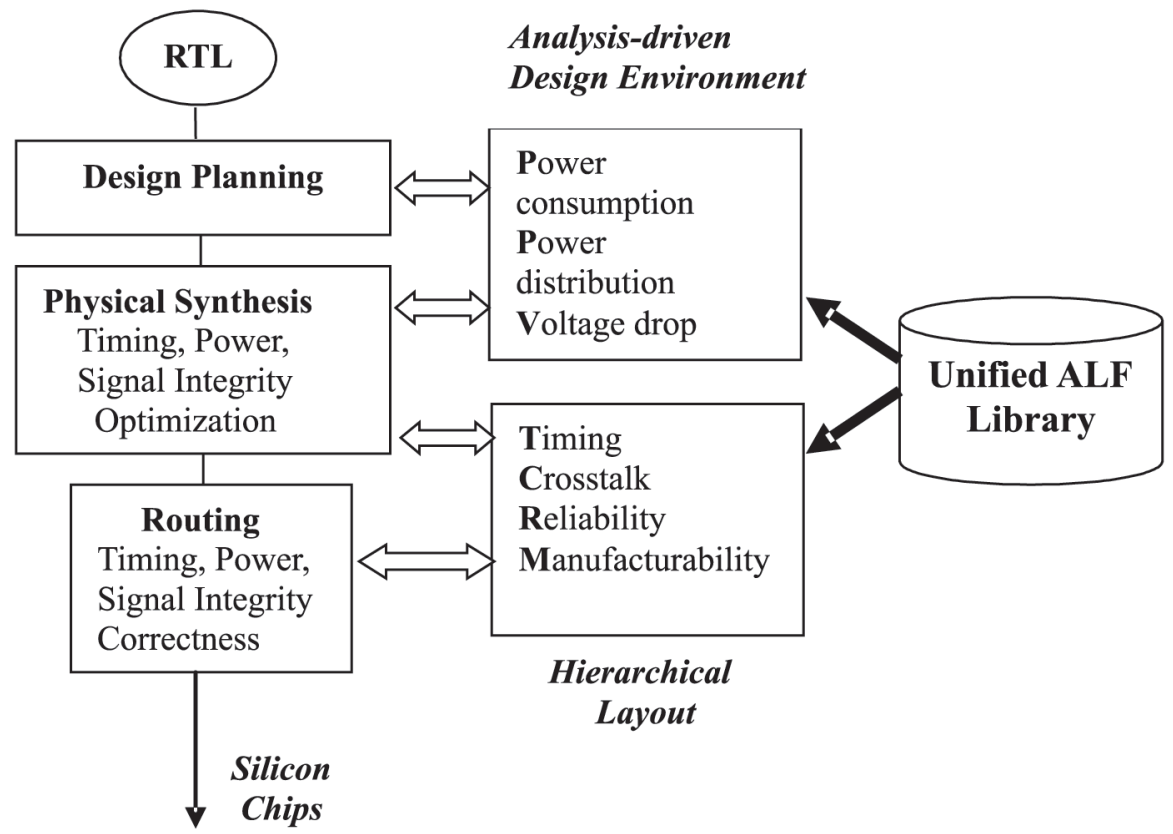

Fig. 2. An Integrated Design Environment Enabled by ALF.

as an elaborate and formalized version of the databook. The purpose here is quite different from hardware description languages such as VHDL, where the language is designed mainly to specify functionality without other aspects of hardware implementation. In summary, the ALF standard paves the way for efficient design environments such as the one depicted in Figure 2. However, the fact that ALF is richer in grammar and semantics when compared to VHDL and Verilog, makes developing parser and compilers for ALF a demanding task.

In the shown environment, an RTL design description is transformed into a netlist by an RTL synthesis tool. The netlist is a collection of cell instances interconnected to form the circuit. The application can use the ALF library to find the library elements needed to map the RTL description into a netlist containing instances of cells. An equivalence checking tool can be used to determine if the RTL-to-netlist transformation has been done correctly, by comparing the RTL design description with the netlist. This application can use the same ALF library used by the synthesis tool. HDL-based simulation tools can be used to verify that both, the RTL design description and the netlist, behave as expected in response to a given stimulus. The simulation tool can use the same ALF cells or higher level ALF modules (for simulation effi- ciency) [10]. During cell placement and interconnect, ALF cell models containing abstract physical information, such as the size and shape of the cell, and the location, size, and shape of the cell pins and routing blockages, which are pertinent for layout, can be used. Also, abstract information concerning implementation details (artwork) within the cell can be represented in ALF; for example, the area, perimeter and connectivity of artwork on specific metal layers. This information is pertinent for manufacturability, such as antenna rules and metal density checks. In addition to cell models, technology rules for routing can also be represented in ALF, such as constraints for the width and length of routing segments, and the distance between vias [10]. In addition to functionality and layout, the ASIC or Integrated Circuit (IC) also has to meet electrical performance constraints such as timing constraints. Other aspects of electrical performance, such as power consumption, signal integrity and reliability, can be modeled using ALF. Also, cell models in ALF support characterization data for timing, power, signal integrity and reliability. After placement and routing, parasitic effects can be extracted and presented in a file using the standard parasitic exchange format. An interconnect model in ALF can describe, using a statistical wire load model (used to statistically estimate parasitics), rules for parasitics estimation based on esti- 
mated routes, or an interconnect analysis model. The interconnect analysis model specifies the desired level of granularity for the parasitics and the calculation of timing, noise, voltage, or current based on instances of parasitics and on an electrical model of a driver cell. The data related to the electrical model of a particular driver cell can be represented in ALF as a part of cell characterization data. The end result is a single and unified library where entities are modeled at the functional, electrical and physical levels. A library that has all timing, power, signal integrity, layout, etc, information allowing for the creation of a system that can compute across any tool precise and consistent parameters.

\section{Design Descriptions in ALF}

ALF consists of several distinguishing characteristics, such as Completeness, Simplicity, Orthogonality, Expressiveness, and Reusability. Descriptions in ALF are complete and selfcontained. The standard supports the complete description of technology data, as well as how that data is to be processed or used by the applications. In contrast, other languages simply allow the presentation of data leaving their interpretations and usage to relevant applications, which may lead to erroneous assumptions. ALF achieves this self-contained characteristic by means of arithmetic models used for calculating mathematically describable quantities; geometric models that describe the shape of physical objects; Boolean expressions to describe the static relationship between objects; vector expressions used to describe temporal change of logic variable and implications [12]. In the following subsections we will introduce the major structural components of this new standard. For the sake of brevity, we will not cover all the details of ALF; readers are referred to $[10,11]$ for an in depth description of the standard.

\subsection{Categories of ALF Statements}

ALF statements are divided into the following categories [10]:

Generic object: the purpose of this category is to provide a definition for use within other ALF statements. Items in this category include, among others, alias declarations, class declarations and group declarations.

Library-specific object: the purpose of this category is to describe the contents of an IC technology library. Items in the category include library and cell declarations, antenna declaration, vector declaration, pin declaration, wire declaration, layer declaration, in addition to other items that are essential to typical IC technology libraries.

Arithmetic model: the purpose of an arithmetic model is to specify a measurable or a calculable quantity within the context of an IC design. Examples of measurable quantities include items such as capacitance, area and delay. An arithmetic model can be a trivial model such as a constant value or an equation or a k-dimensional look-up table.

Arithmetic model container: the purpose of the container is to provide a context for an arithmetic model. For example, the purpose of the arithmetic model container limit is to specify one or more quantifiable design limits.

Geometric model: the geometric model is used to describe the form of a physical object used in the physical design of an IC. Geometric model identifiers include polylines, dots and rings that are used in physical IC designs.

Annotation and Annotation container: the annotation provides a qualifier or a set of qualifiers for an ALF statement, while the container provides a context for an annotation.

Auxiliary statement: the auxiliary statement provides an additional description within the context of a library-specific object, an arithmetic model or geometric model.

An ALF statement can itself contain one or more other ALF statements. The former is called the parent of the latter and the statements form a parent-child relation ship. For example, within the library-specific-object category, a sublibrary can be the child of a library and the sublibrary can be the parent of a cell. Figure 3 is an example of the parent-child relationship amongst library specific objects [10]. The figure also depicts ALF design domains.

Although the strength of ALF lies in its domainindependent language elements, such as templates (for re-usable library descriptions) and arithmetic models, the language elements can 


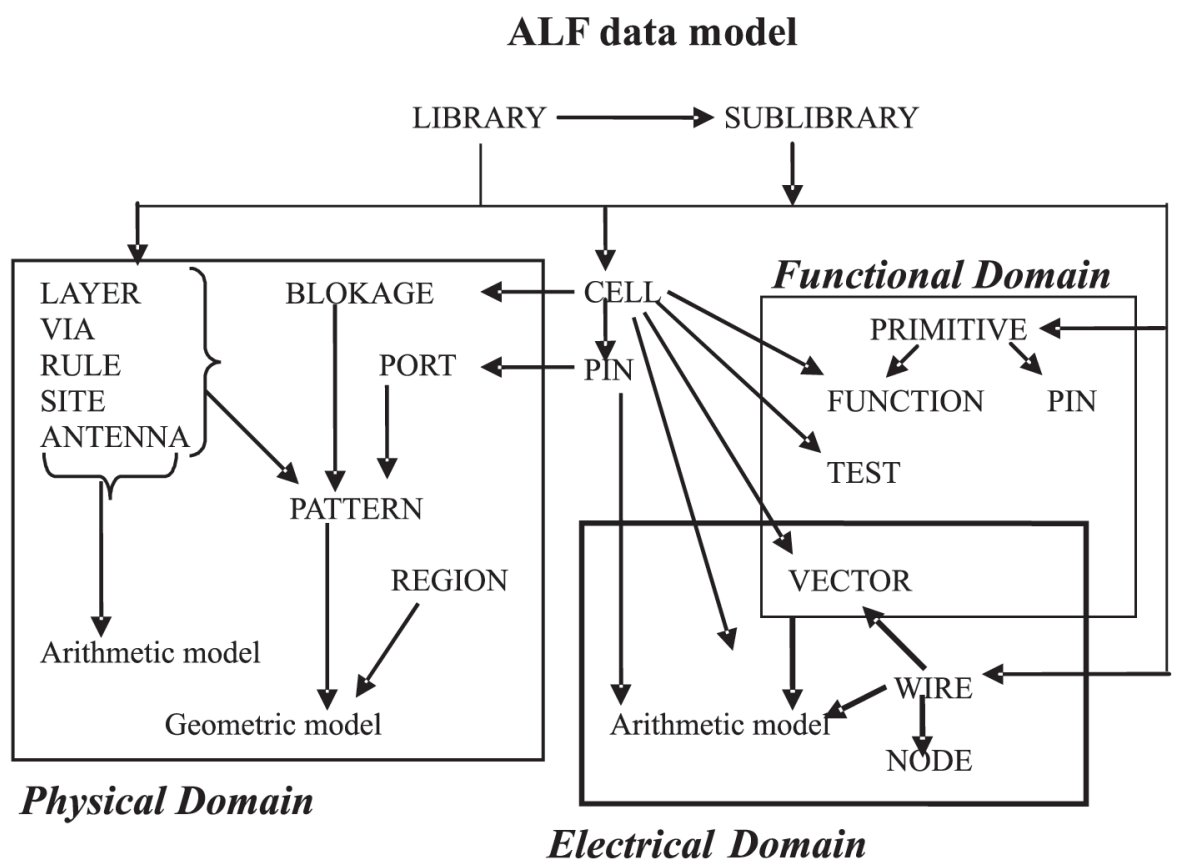

Fig. 3. ALF Domains and Child-parent Relationships.

still be associated with modeling domains as shown in Figure 3.

ALF is a simple, yet very expressive language with only a few basic syntax rules. Therefore, translating existing technology libraries into other formats to ALF is straightforward and simple, provided the precise interpretation of other library format data is well understood. Syntactical keywords in ALF are written English words, which have been in use and are therefore familiar to those in the industry (e.g. SIGNALTYPE, ANTENNA, VOLTAGE, CURRENT).

ALF allows annotations to be used in specific contexts for readability and to convey information giving the appearance of familiar 'Data book' look to the library. For example, an application may infer the behavior of a cell with annotation 'celltype =memory', without analyzing its functionality. Annotations are so powerful that they can even specify whether a cell is usable for a particular application. For example, a cell with annotation 'restrict_class=synthesis' is meant only for synthesis tools, and may not be used by layout tools. ALF provides a great versatility by introducing Template Objects. A template object allows the re-use of a certain behavior or logic element regardless of whether it can be interpreted as a conventional HDL module or not. It introduces the concept of instantiating not only modules, but behavioral blocks and even logic nodes such as PIN and WIRE. Basically, a user can define any ALF object as a Template together with all the physical, electrical, layout, etc. characteristics and re-use this pre-defined object throughout the design in the form of a template instantiation. A template instantiation gives further control to ALF users by introducing the concept of Place Holders in order to customize the internal attributes of the particular object. Not only the individual logic nodes, but also behavioral blocks, can be re-used using templates. By having placeholders inside the template behavior, the designer is given the opportunity to define the behavior generic to the names of particular logic nodes (wire, pin, etc) that take part in the behavior. The template instantiation assures that designer's custom logic nodes are taking part in the behavior that is re-used as a template library object. This is a dramatic improvement when compared to the conventional HDLs. Simply, the designer can use many predefined objects and also import them from third parties. Design becomes abstract-contracted and more organized. It adds up a great value to the digital design flow when using ALF. 


\subsection{Examples of Characterization and Modeling using ALF}

ALF can be used to describe the functional, electrical, performance and layout characteristics of an ASIC or Silicon-on-Chip technology library that is scalable from simple cells to complex macro modules. As discussed earlier, an ALF library can be part of any ASIC or IC implementation flow that uses cells as building blocks. A specification of a cell in ALF includes its name, type, functionality, pins, besides additional cell items. The cell type, for example, can be of type buffer, latch, memory or core. An example is given below:

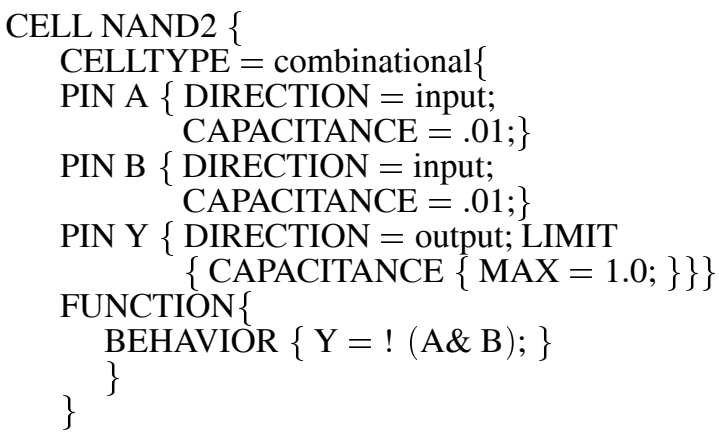

Arithmetic models in ALF are very descriptive and the calculations can be described as:

(a) single number (trivial_arithmetic_model),

(b) an equation (equation_based_arithmetic_ model),

(c) in table form (table_based_arithmetic_ model), or

(d) a nested arithmetic model.

For example, a trivial arithmetic model may describe static capacitance as:

"CAPACITANCE $=2.3$ UNIT = 1e-15;,"

An equation_based_arithmetic_model can describe VOLTAGE and TEMPERATURE dependent capacitance as:

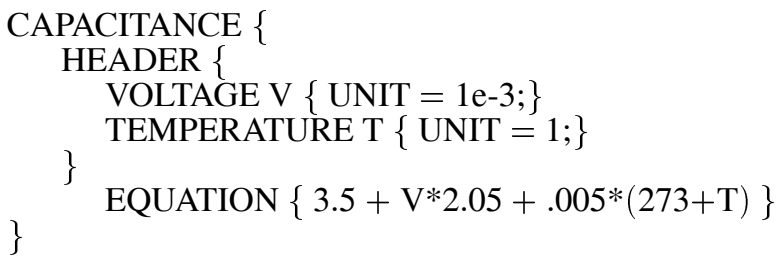

or by a table_based_arithmetic_model as:

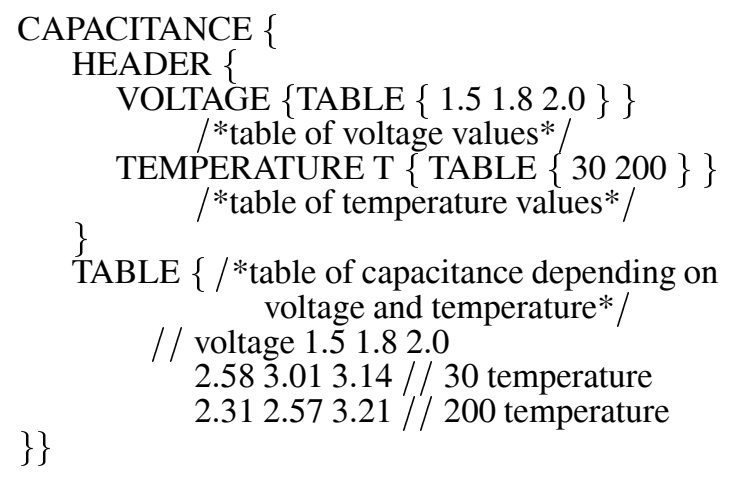

Information, such as calculation type, interpolation methods of table entries, minimum- maximum limits and units, can be given with annotations, so that it is 'self documenting'. Apart from pre-defined arithmetic models and the associated annotations, user can define his own models and annotations using the KEYWORD declaration.

As discussed earlier, a template instantiation allows for re-use of logic nodes and behavioral blocks. For example, a single template associated with PIN can be reused to define different pins whose properties are different from each other.

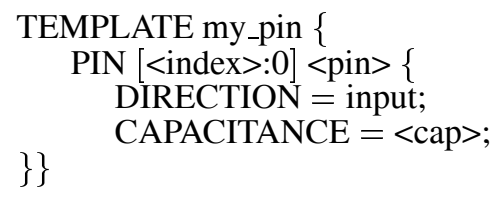

Instantiation of above template may be static or dynamic as follows:

my_pin $\{150.5\}$ or my_pin $\{$ index $=15$; cap $=0.5\}$

$$
\text { /* a } 16 \text { bit pin with } .5 \text { capacitance */ }
$$

my_pin $=$ dynamic $\{31$ cap_val $\}$

$$
\begin{aligned}
& \text { /* a } 32 \text { bit pin whose capacitance 'cap_val' } \\
& \text { is determined at runtime by the application */ }
\end{aligned}
$$

If there are common characteristics within ALF statements, those commonalities can be defined only once, rather than repeating them for each element by grouping them to one set. In ALF, 
this concept is allowed with the GROUP statement to improve the efficiency and compactness.

For example, a set of characteristically equivalent pins can be defined, as shown below, as in G1 instead of G2.

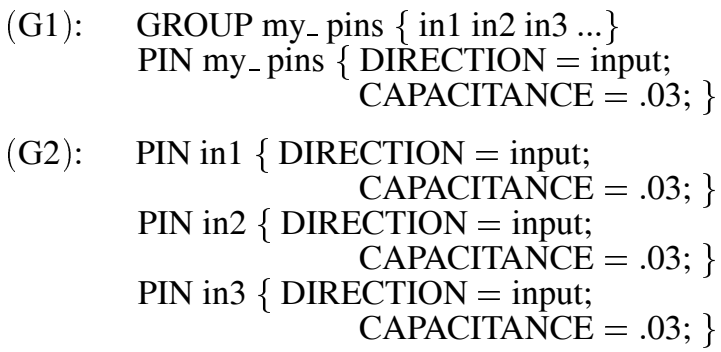

(G2): $\quad$ PIN in $1\{$ DIRECTION = input; CAPACITANCE $=.03 ;\}$

Also, Grouping can be used to convey that a certain characteristic is common for a set of elements. For example, the UNIT of time measurement of DELAY, SKEW and SLEWRATE can be expressed as nano seconds (ns) only one time, rather than defining it in each model, using the two statements below:

\section{GROUP time_unit \{ DELAY SKEW SLEWRATE $\}$ time_unit $\{$ UNIT $=$ ns; $\}$}

The vector expression concept that ALF introduced is very powerful in modeling signal changes with associated data measurements. It allows abstraction of modeling views at any hierarchy level, far beyond simple library cells. Vector expressions can describe an event or an event pattern to be associated with a particular set of data or an arithmetic model. For example, vector expressions within the context of VECTOR can model DELAY, POWER, ENERGY, etc., associated with an event sequence described by the vector expression.

In a 2 -input AND (out, in 1 , in2) gate, if a $0 \rightarrow 1$ transition on in 1 input and $1 \rightarrow 0$ transition on in 2 input occur before the input-to-output delay elapses, a glitch may result at the output pin. It is possible to describe the glitch ENERGY by a higher order vector as:

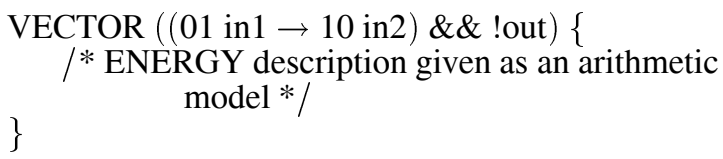

Vector expressions, being event sequence-dependent, offer modeling capability for asynchronous behavior, as well as event synchronization. Although ALF stands for Advanced Library Format, it is powerful enough to describe and model high level behaviors and complex systems. It supports constructs to express the functional behavior in either procedural or concurrent flow. Vector expressions within the context of BEHAVIOR can model higher order sequential logic, which describes sequences of logical events or transitions in addition to static logical states. As a simple example, the event sequence described by ' 01 in $\rightarrow 10$ out' will be true at the instant of time when a rising edge on 'in' is followed by a falling edge on 'out'. Vector expressions can be extended to describe any complex event pattern using boolean and vector arithmetic provided in the language, which is a facility that the behavior can be expressed in a more convenient way, compared to expressing with a set of level_sensitive and edge_sensitive sequential logic. This expressiveness allows complete characterization of macro cells or IP blocks with abstraction to hide sensitive implementation information.

ALF facilitates reusability with inheritance where a model can implicitly inherit arbitrary properties from a different model of the same ALF type. This is another feature for non- repetition of the same characteristic over and over within each and every context. In the following example, UNIT within a DELAY model defined in the global level of the library can be inherited by a DELAY model within a VECTOR, if the UNIT is not specified in the latter.

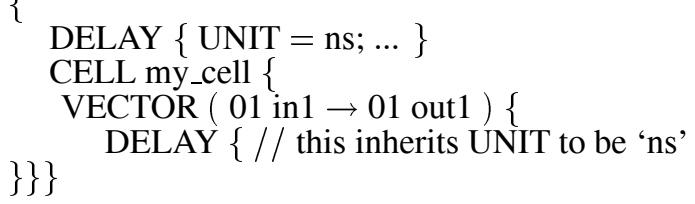

Also, ALF facilitates explicit inheritance with CLASS statement. In the following example, PIN 'my_pin1' inherits only SIGNALTYPE and POLARITY annotations, while 'my_pin2' inherits all three annotations: SIGNALTYPE, POLARITY and PINTYPE.

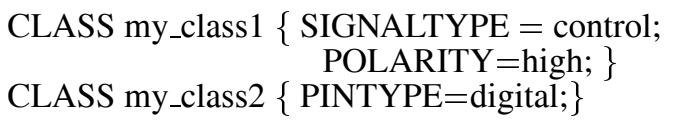


PIN my_pin1 \{ DIRECTION=input; CLASS $=$ my_class $1 ;\}$

PIN my_pin2 $\{$ DIRECTION=input; CLASS $\{$ my_class 1 my_class 2$\}\}$

ALF also introduces types of pre-defined primitives in addition to the ones available in standard HDLs. For example, ALF_FLIPFLOP, ALF_LATCH, ALF_MUX and so on. These allows users to build complex structural functional modeling where it would be a tiring job to model with primary language constructs of other standard HDLs.

ALF supports definition of new key words, their contexts/scope and optional valuetype and values which allowed to be used in other ALF statements. This is one aspect of extensibility of ALF. For instance, the user can define an arithmetic_model 'my_model' in the context of VECTOR whose value type is a number as shown below.

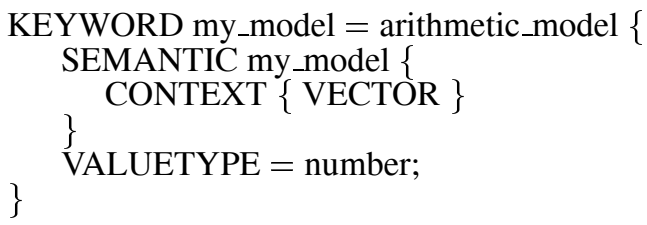

Arithmetic models can also be used, for example, to specify the power consumption of cells or the entire circuit. The semantics below describes an arithmetic model of power and energy.

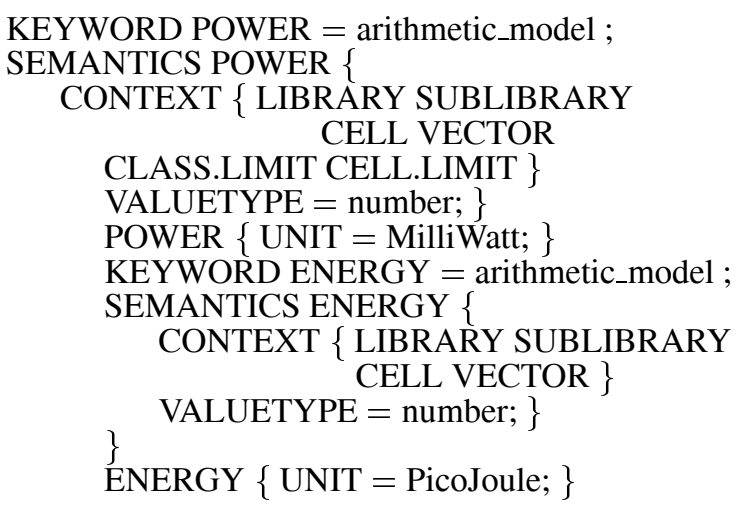

The arithmetic model container limit can be used to specify a design limit for power consumption.

ALF also supports interconnect modeling and can characterize cell delays using a distributed
$\mathrm{RC}$ load. It also supports interconnect delay and noise calculation. Arithmetic models may also be used to quantify the hot electron effects. The purpose of the hot electron calculation is to evaluate the damage done to the performance of an electronic device due to the hot electron effect. Here, Flux and/or Fluence models will be in the context of a CELL or within a VECTOR. Total fluence or flux of a cell will be calculated by combining the data of all models within the CELL or the VECTORS within the cell. Design changes can then be implemented to limit the hot electron effect with minimal disturbance to other design parameters.

In ALF, timing models of cells consist of two types: delay models for combinational and sequential cells, and timing constraint models for sequential cells. Both types can be described using timing arcs. A timing arc is a sequence of two events that can be described by a VECTOR expression "event $\mathrm{e} 1$ followed by event e2". For example, a particular input to output delay of an inverting logic cell is identified using the following timing arc:

$$
01 \mathrm{~A} \rightarrow 10 \mathrm{~F}
$$

which reads "rising edge on input A is followed by falling edge on output F". A set up constraint between data and clock input of a positive edge triggered flip-flop is identified using the following timing arc:

$$
01 \mathrm{D} \rightarrow 01 \mathrm{CP}
$$

which reads "rising edge on input $\mathrm{D}$ is followed by rising edge on input CP". In addition, ALF allows designers to define a delay arithmetic model to specify a time interval, implying a causal relationship between two events. Users can also make use of the powerful modeling capabilities of ALF to develop arithmetic models for parameters such as SLEWRATE, SETUP and HOLD times.

\section{Conclusion}

The ALF standard has been under development for the last few years, resulting in a library format that will soon enjoy widespread support within the industry. In this paper the primary goals and benefits of the standard are discussed. 
The basic principles of modeling and characterization in ALF are introduced, and a subset of its dense set of features is presented. The standard meets the critical need of IC manufacturers to be able to include various characterization data without library format constraints. With its comprehensive modeling capabilities and extensibility, combined with EDA tools that analyze ALF based technology libraries and designs, ALF is posed to be the best modeling language to be used in developing high-end Soc and ASIC technical design libraries.

\section{References}

[1] Deep-Submicron Signal Integrity: Issues, Analysis and Solutions, White Paper, Magma Design Automation Corp., March. 2004.

[2] C. Bittlestone, A. Hill, V. Singhal And A.N.V. Architecting ASIC Libraries and Flows in Nanometer Era, IEEE/ACM Design Automation Conference, June 2003, Anaheim, California.

[3] M. SMITH, Application-Specific Integrated Circuits, Addison-Wesley, 1997.

[4] W.K. CHEN, The VLSI Handbook, IEEE Press, 1999.

[5] H. Bhatnagar, Advanced ASIC Chip Synthesis Us ing Synopsys Design Compiler, Physical Compiler and Prime Time, Kluwer, 2002.

[6] M. Karunaratne, A. Sagahyroon AND W. WEERAKODY, An Advanced Library Format for ASIC Design, Proceedings of the IEEE Canadian Conference on Electrical and Computer Engineering, 2004.

[7] T. BEDNAR ET AL, Technology migratable ASIC library design, IBM Journal of Research and Development, vol. 40, no. 4.

[8] W. Roethig ET AL, Signal Integrity on $300 \mathrm{Mhz}$ SoC Design using ALF Libraries and Tools, EDA Vision, May 2002.

[9] J. ABRAHAm AND S. Churiwala, Flexible Model for Delay and Power, www.si2.org/ola/ pdf/olawhitepaper.pdf

[10] IEEE std. 1603-2003, A Standard for an Advanced Library Format (ALF) Describing Integrated Circuits Technology, Cells, and Blocks, IEEE, 2003.

[11] W. Roethig, Accellera ALF Tutorial, IEC Workshop, Oct. 2002.

[12] W. Roethig, Coherent functional, electrical and physical modeling of IP blocks using ALF, Proceedings of the IEEE Custom Integrated Circuits Conference, 2001.
Received: December, 2004

Accepted: April, 2005

Contact address:

Dr. Assim Sagahyroon

College of Engineering

American University of Sharjah P.O. Box 26666 Sharjah, UAE

Phone: +97165152952

e-mail: asagahyroon@ausharjah.edu

DR. ASSIM SAGAHYROON is an associate professor with the Computer Engineering Dept. at the American University of Sharjah. He received his MSc from Northwestern Univrsity and the PhD from the University of Arizona. His research interests include VLSI design automation, computer architecture, embedded systems and fuzzy logic applications in engineering.

DR. MADDU KARUNARATNE is an assistant professor at the University of Pittsburgh at Johnstown. He received his PhD from the University of Arizona. He specializes in design automation, VLSI testing, fault modeling, and power estimation. He has over 12 years of extensive industrial experience as a consultant in the Silicon Valley. 